\title{
MADS-Box Transcription Factor FRUITFULL Orthologs in Nicotiana Promote Transition to Flower, but Did Not Significantly Alter Capsule Development
}

\author{
Abeer Mohamed \\ Department of agricultural botany, faculty of agriculture (Saba Basha), Alexandria University \\ Corresponding author: abeer.mohamed@gmail.com
}

\section{ABSTRACT}

Understanding the genetics mechanisms of flower and fruit development is very crucial for efficient breeding of many agronomical important crops. MADS-box group of transcription factors has been known for its significant contribution of controlling fruit development. FRUITFULL $(F U L)$ is one of the genes that plays important roles in this regard. For more understanding of FUL gene function in capsule dry-fruit development, this study used virus Induced Gene Silencing method to knockdown FUL gene expression in the newly developed model plant, Nicotiana obtusifolia. Treated plants exhibited delayed flowering, shorter inflorescence stems and longer fused sepal when compared to wild type and control group. However, no altered fruit phenotypes were observed among the treated $N$. obtusifolia plants. Downregulation of FUL gene in Nicotiana obtusifolia, allows us to assess a better understanding of its function in dry fruit species of the family Solanaceae. It also suggests that FUL protein plays different roles in the development of dry versus fleshy fruits in Solanaceae.

Key words: Nicotiana obtusifolia, FRUITFULL gene, Flower development.

\section{INTRODUCTION}

Fruit is a very important organ for plant as it mediates maturation and dispersal of the seeds. Understanding the genetics mechanisms of flower and so fruit development is very crucial for efficien breeding of many agronomical important crops. One gene heavily implicated in fruit development is AGAMOUSE-LIKE 8 (AGL8) or FRUITFULL $(F U L)$. This gene is one of the MADS-box group of transcription factors. FUL gene function has been studied intensively in Arabidosis thaliana in which ful mutants show improper fruit development (Gu et al, 1998; Ferrándiz et al, 2000a; Ferrándiz, 2002). Although floral organ identity is not affected in Arabidopsis ful mutant, FUL gene found to act redundantly with APETALA1 (AP1) and CAULIFLOWER (CAL) genes to promote transition to floral meristems (Ferrándiz et al, 2000b). Arabidopsis FUL with SUPPRESSOR OF OVEREXPRESSION OF CONSTANS 1 (SOC1) gene have been shown to regulate flowering time, affect determinacy of all meristems and plan longevity (Smykal et al, 2007; Melzer et al, 2008). Overexpression of Arabidopsis FUL in Brassica juncea (Østergaard et al, 2006) and Antirrhinum FUL ortholog, DEFICIENS-homolog28 (DEFH28), in Arabidopsis (Müller et al, 2001) affect lignification process and carpel wall development FUL tomato orthologs, SIFUL2, regulate the expression of ripening-related genes (Bemer et al, 2012; Wang et al, 2014). FUL gene also has assigned a role in tomato leaf development (Burko et al, 2013). As with many crops produce capsule dry fruit, examining the role of FUL in capsule fruitbearing species and studying whether $F U L$ gene has similar role in capsule fruit species as in Arabidopsis (silique fruit) should increase our understanding of FUL gene function in fruit development. This study aims at studying the function of a FUL orthologs in capsule dry fruitbearing species Nicotiana obtusifolia, which was chosen for many reasons including compatible size, ability to flower and grow faster compared to other Nicotiana varieties. $N$. obtusifolia is a member of the Solanaceae family which become vital to human in multiple ways. Genera such as Datura and Atropa have been shown to have anticancer and antiasthmatic properties (Chiarini, 2009; Soni et al, 2012). In addition, Solanaceae species such as tomato and eggplant are increasingly important component in human diet (Chiarini, 2009). Determining the roles of FUL genes in dry versus fleshy fruits contributes to the understanding of the gene regulatory network behind ripening. VirusInduced Gene Silencing (VIGS) method is used in this study to specifically silence or reduce the expression of FUL2 gene in $N$. obtusifolia, NobFUL2, through Post-Transcriptional Gene Silencing mechanisms (PTGS) (Dinesh-Kumar et al, 2003). Tobacco Rattle Virus (TRV) vector sequence is one of the widely used in VIGS (Bachan and Dinesh-Kumar, 2012). It composed of two separate components (vectors): TRV1, which carries 
movement and coat proteins and is not altered in the VIGS procedure, and TRV2, from which the virulence genes replaced by a fragment of the target gene to initiate an immune response. Upon infection of virus to the plant, synthesis of viral double stranded RNA (dsRNAs) lead to the activation of the antiviral RNA silencing pathway and the subsequent knockdown of the endogenous host gene (Ding and Voinnet, 2007). The silencing signal spreads systemically through the phloem system of the plants (Kalantidis et al, 2008). Compared to other techniques to knock down genes, VIGS has the advantages of being rapid tool that does not need stable plant transformation. It can be used in wide range of plant system (dicot or monocot) (BurchSmith et al, 2004; Robertson, 2004; Aly et al, 2009) with relatively low cost.

\section{MATERIALS AND METHODS}

\section{Plant Materials and growth conditions:}

Nicotiana obtusifolia seeds were obtained from third generation of accession TW143, US Nicotiana Germplasm collection. Seeds were germinated and continue to grow on soil in 2.5 inch pots under 12 hours light regime.

RT-PCR

The expression of Nicotiana obtusifolia FRUITFULL 2 (NobFUL2) gene was assayed in cauline and rosette leaves, young bud, pre-anthesis bud, 4dpa fruit and $7 \mathrm{~d}$ pre-dehiscence. Total RNA from tissue samples was prepared using Trizol reagent (Invitrogen) and then was subsequently treated with DNaseI (NEB). $1 \mu \mathrm{g}$ of RNA was used for cDNA synthesis with SuperScript III (Invitrogen). Forward Primer 5'GGTGAAAGAAAGGGAGAAAGA-3' and reverse primer 5'-TATCCAAGGCGAGGATGATA-3' used to amplify $425 \mathrm{bp}$ from NobFUL2. Reactions were run for 34 cycles at an annealing temperature of $58^{\circ} \mathrm{C}$. ACTIN was used as endogenous control.

\section{Quantitative RT-PCR (qRT-PCR)}

To test the down-regulation of NobFUL2 in VIGS-treated plants, RNA was extracted from rosette leaves and pre-anthesis bud tissue. Total RNA was prepared using RNeasy kit (Qiagen) and subsequently treated with DNase (NEB). Total RNA $(1 \mu \mathrm{g})$ was used for cDNA synthesis with SuperScript III (Invitrogen). Leaf cDNA was diluted 1:5 and bud cDNA samples were diluted 1:20. PCR product was amplified using locus-specific primers designed using Primer ExpressTM version 3.0 (Applied Biosystems) Forward primer 5'TCGTCTACGGTTAGGAAGAATATG $3^{\prime}$ and reverse primer GGCGAGGATGATATGGTACTACATTA

Down-regulated samples were analyzed relative to Independent wild-type samples using $\Delta \Delta \mathrm{Ct}$ method of analysis.

\section{TRV-VIGS}

A 425 bp fragment of NobFUL2 which include the $\mathrm{K}$ and $\mathrm{C}$ domains of the protein was amplified from inflorescence cDNA using forward primer,5'CGACGACAAGACCCTtactgctactg 3' and reverse primer; 5'GAGGAGAAGAGCCCTcatattcttcet 3' that introduce compatible ends (uppercase letters) and so facilitate cloning of NobFUL2 fragment into TRV2 LIC vector as described in Dong, et al, 2007. Followed by Agrobacterium tumefaciens transformation and cultivation on selective LB medium. The cultures were then mixed in a $1: 1$ ratio, Infiltration of seedling with only three leaves was performed after 19-22 days of seed sawing. At the time of infiltration, some seedling left untreated (labeled WT), and the rest infiltrated with either TRV2-empty (labeled empty) or TRV2-NobFUL (labeled FUL). Physiological observations and data collection started from the day of infiltration and extended until fruit dehiscence. ANOVA calculations were performed on measurements between WT, empty vector, and VIGS-NobFUL2 plants.

\section{RESULTS AND DISCUSSION}

NobFUL2 spatial gene expression is similar to Tomato FUL genes:

With the exception of the intense studies into Arabidopsis fruit development, most fruit developmental studies have primarily focused on fleshy species Tomato for their essential use in human diet (Giovannoni, 2004). As a consequence, few studies have investigated the FUL gene expression in dry-fruits species. In Nicotiana sylvestris only one ortholog of the FUL gene was identified (Jang and An; 1999) and another FUL ortholog, NtMADSII, was described in Nicotiana Tabuccum (Jang et al, 2002). On the other hand, four FUL paralogs in tomato were studied (Hileman et al, 2006; Bemer et al, 2012; Burko et al, 2013). NobFUL2 protein contains Lys $^{51}$ and Asp ${ }^{60}$ characteristic of the MADS domain of the SQUA family and does not have CaaX-box, but encodes for the polypeptide "MPQWMLR", similar to Arabidopsis FUL gene (data not shown). NobFUL2 protein share $97 \%$ identity to Nicotiana FUL, NtMADS11 (accession No. AAO12211.1) and NsMADS1 (accession no. NP_001289508.1). NobFUL2 also share $92 \%$ identity to Tomato FUL2, SIFUL2 (accession no. NP_001294867.1) and 82\% identity to SIFUL1 (TDR4; accession No. AAM33098.1). Therefore it was given the name NobFUL2. In the current study, NobFUL2 gene found to be expressed in vegetative organ, rosette leaves. The transcript was present at much higher level at the young floral bud and mature flower stages compare to young fruit (Figure1). This result indicates that it functions in both reproductive and vegetative organs. Similar spatial expression results 
have been reported with NsMADS2, NtMADS11 and predicted their role in floral induction process and the maintaining of the reproductive phase (Jang and An, 1999; Jang et al, 2002). Similar results have been reported with the expression of the Tomato orthologs, SlFUL1 and 2, as high in flowers and throughout fruit development, as well as low leve transcript in leaves, indicating role in leave development, floral induction and fruit development, particularly in shape, pigmentation, and, most notably, pericarp thickness, which is feature that differentiates dry and fleshy fruits (Bus et el, 2003; Bemer et el, 2012; Burko et el, 1013; Wang et el, 2014).

\section{VIGS treatment causes NobFUL2} downregulation in leaves and floral bud tissue:

After using VIGS to knockdown NobFUL2 gene, it was very important to determine the level of downregulation if any in all treated plants. Therefore RT-PCR was conducted for all 70 NobFUL2 infiltrated plants. All showed successfu down-regulation in comparison with wild type (WT) and the TRV2 empty vector-treated plants (data not shown). As more quantified step, qRT-PCR were performed on seven individuals selected randomly from the VIGS-treated plants (Figure 2-A). The samples were taken two weeks after treatment to give sometime for the virus to spread across all leaves and stem tissue. Compare to the WT, NobFUL2 expression level in treated-plants leave tissue was $70 \%$ less (Figure2-A). Considering the effect of NobFUL2 downregulation on flowering time and development, re-checking of NobFUL2 expression level in the bud tissue was necessary step to evaluate and discuss the outcome of VIGS treatments. As expected, the level of NobFUL2 gene expression in bud tissue was not the same as in leaves tissue of the same plant (Figure2-A, B). In two samples of bud tissue (1A and 6A; Figure 2-B) NobFUL2 expression was $85 \%$ less compare to WT and was even less than NobFUL2 transcript in leaves. Three samples, 5A, 57A and 79C, have shown expression equal to $70 \%$ less than WT and similar to the gene expression level in leaves. Two samples show higher level of NobFUL2 transcrip than its counterpart in leaves, but the expression level was still less than WT by $50 \%$. QRT-PCR data show down regulation of NobFUL2 gene expression in both leaves and bud tissue which confirm that the phenotype we are getting is correlated with the NobFUL2-VIGS treatment is due to the downregulation of NobFUL2 and that the downregulation was exist in the bud tissue as well as in leaves. Yet is mosaic, but mostly the expression was $70 \%$ less than untreated plants (Figure 2-A, B)
Downregulation of NobFUL2 causes delayed flowering and shorter inflorescence stem:

Nicotiana obtusifolia plant grows and develops as a ring of rosette leaves, followed by elongating an inflorescence stem (bolting), and then flowering. The timing of these developmental events in response to down-regulation of NoFUL2 was observed and compared with observations of wild type and empty vector-treated control plants. Flowering time, is one of the well-known function of the FUL gene (Jang et al, 1999; Pabón-Mora et al, 2012; Ferrándiz and Fourquin 2014). Therefore, flowering time was the first observation to be recorded. In order to study and quantify the differences in flowering time, many observation have been recorded on treated, control and WT plants, including the time of bolting, measured as the time from germination to elongation of the inflorescence when reach $1 \mathrm{~cm}$ long. Other significant phenotypes were the number of rosette leaves at bolting, the number of days to anthesis (opening) of the first flower. Down-regulation of NobFUL2 resulted in delayed bolting when compared to wild type and empty vector-control plants $(\mathrm{p}<0.001)$ (Figure 3). As shown in Figure 3, VIGS- NobFUL2 treated plants bolts at $51.2 \pm 2.4$ days with more than 10days delay compare to WT which bolt at $41.5 \pm 1.7$ days and control group (E) which bolts at 43 days. Another indication of delay in flowering time was shown by the delay in the time of anthesis of the first flower. VIGS-NobFUL2 treated plants have their first flower to open at anthesis at $71 \pm 3$ days with more than 13 days delay compare to WT which start anthesis at $56 \pm 3.4$ days and empty-group plants to start anthesis at $58 \pm 0.4$ days (Figure 3). The number of rosette leaves at bolting (Figure 4) was also counted as additional measures of flowering time. NobFUL2 downregulated plants had significantly more rosette leaves, $15.8 \pm 1$ leaves, when compared to that of wild type, $10.7 \pm 0.9$ leaves, and empty vector plants with 12 \pm 1 leaves (Figure 4). According to the present finding, NobFUL2 gene plays a role in promoting floral transition and so affecting flowing time. This finding has been indicated before in other Nicotiana species and Arabidopsis (Jang et al, 2002; Smykal et al, 2007; Ferrándiz et al, 2000b). A variety of other phenotypic abnormalities recorded, including significantly shorter inflorescence stems of VIGS-treated plants, $30 \pm 3.3 \mathrm{~cm}$, compare to the WT and empty vector plants which reach $36.9 \pm 2$ and $35.9 \pm 2.9 \mathrm{~cm}$ long respectively $(\mathrm{p}<.001$; Figure $5)$. This results were correlated with the finding that average internode lengths of the VIGS-NobFUL2 treated plants were significantly shorter than WT and empty vector plants (data not shown) Additional abnormal phenotypes were recorded and have not been indicated in any previous study; 


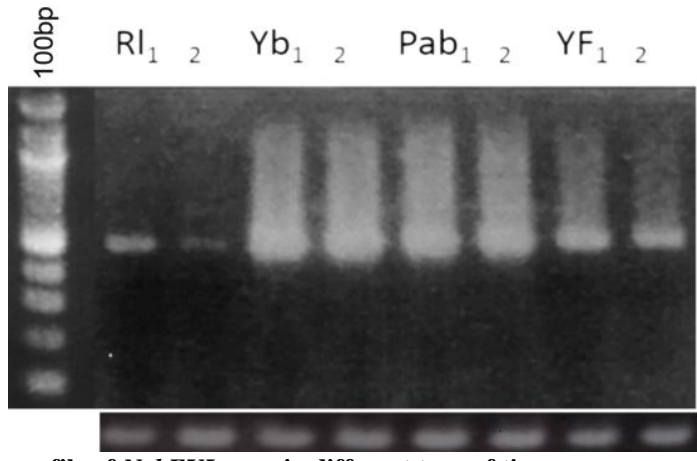

Figure 1: Expression profile of NobFUL gene in different type of tissue.

From a wildtype (WT) N.Obtisofolia, different tissue samples were collected from two individual plants; Rosette leaves (RI), Young bud (Yb), Post-anthesis bud (Pab), Young fruit (YF). The level of NobFul2 gene expression is shown. Actin expression was used as an endogenous control (bands at the bottom)

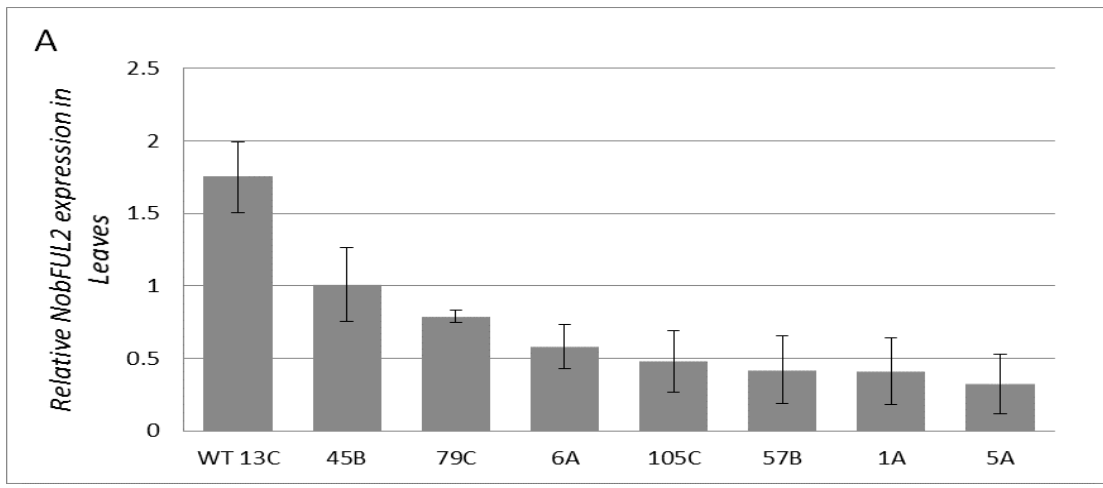

B

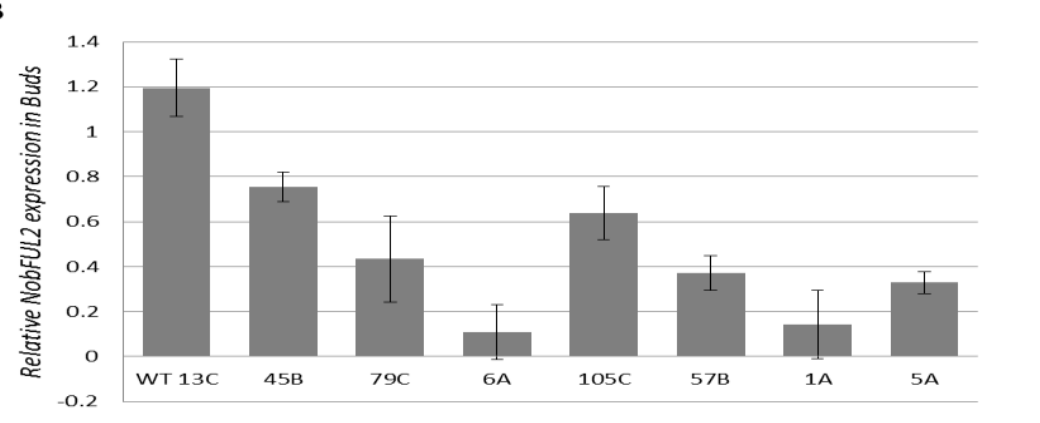

Figure 2: Down-regulation of NobFUL2 in Rosette leaves and Buds of VIGS-treated plants.

(A) Quantitative RT-PCR using cDNA prepared from leaves of VIGS-treated plants showing the fold change in NobFUL2 expression relative to wild-type leaves in seven plants (numbered). (B)Quantitative RT-PCR using cDNA prepared from pre-anthesis buds of VIGS-treated plants that showed downregulation of nobful 2 in leaves. Values are means. SD for three technical replicates. $N$. Obtusifolia Actin was used as endogenous control. 


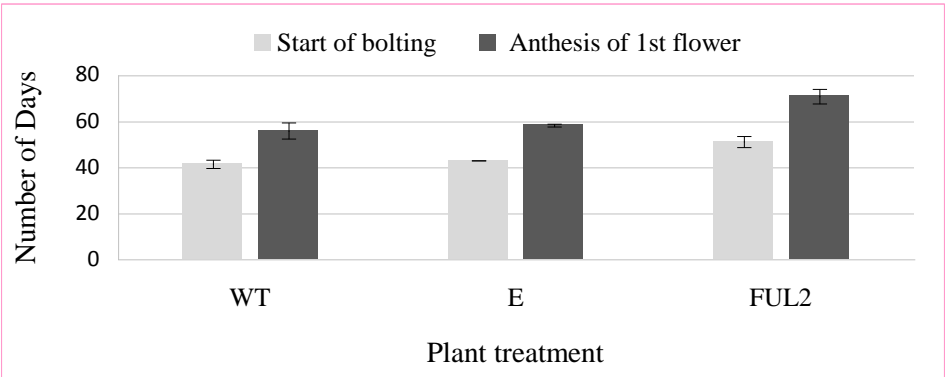

Figure 3: Time for bolting and anthesis of first flower.

Number of days was counted from germination until bolting and until anthesis of first flower. Some seedlings were kept without treatment (WT), some were infiltrated with empty TRV2 vector (E) and some were infiltrated with TRV2 vector containing 425bp of NobFUL2 gene (FUL2). The difference between controls and NobFUL2 plants is significant $(\mathrm{p}<.001)$ as measured by ANOVA analysis of significant differences.

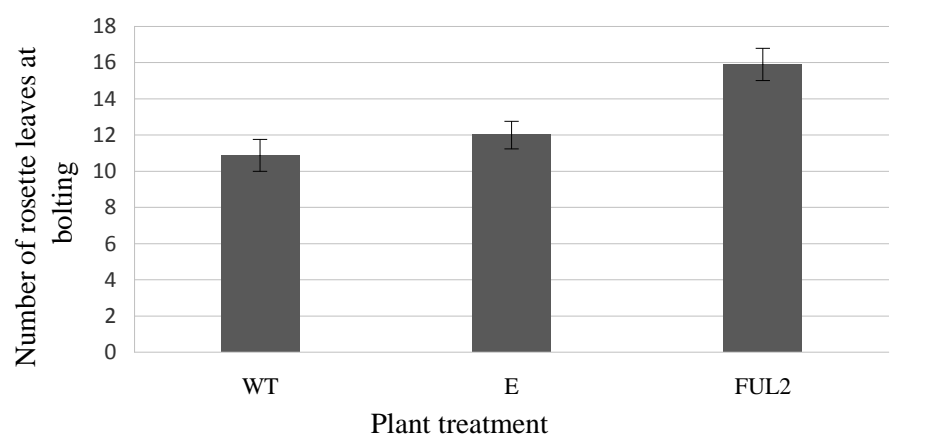

Figure 4: Variation in the number of rosette leaves at the time of bolting.

The number of rosette leaves at the time of bolting for wild type (WT), empty vector (E), and NoFUL2 VIGS-treated plants. The difference between controls and NoFUL2 plants is significant $(p<.001)$

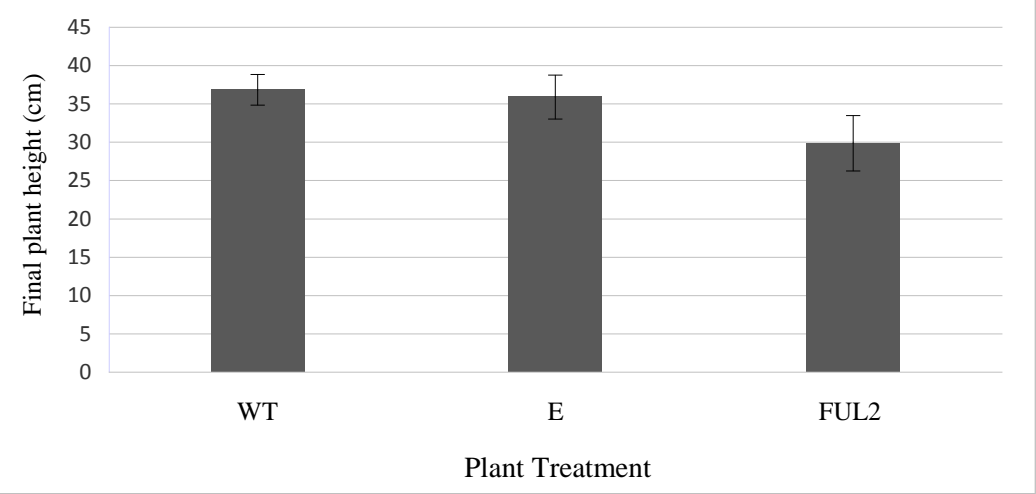

Figure 5: Final inflorescence height of VIGS-NobFUL2 treated plant compared to WT and E plants: Inflorescence stem height is significantly shorter upon NobFUL2 down-regulation when compared to WT and empty-vector (E) plants. 

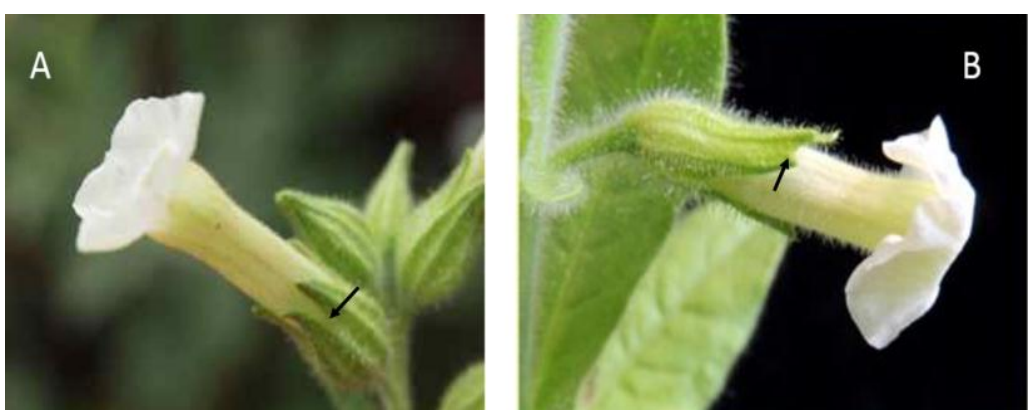

Figure 6: Flower sepal morphology.

(A) Showing a flower at anthesis of the wild type $N$. obtusifolia with normal unfused sepal. (B) is showing a flower at anthesis of VIGS-NobFUL2 treated plant with longer fused sepal.

increased branching (data not shown) and longer fused sepals (Figure 6) were observed in treated plants. No abnormal developmental of morphological phenotypes were observed in the fruit structures and the timing of fruit dehiscence. That is in contrast to what was expected. Previous studies indicate that the FUL genes in Nicotiana and Arabidopsis have equivalent roles inhibiting frui dehiscence and so affecting fruit development (Smykal et al, 2007; Ferrándiz and Fourquin, 2014) In Tomato fleshy fruit, upon down-regulation of SIFUL1 and SlFUL2 fruit coloring and ripening were impaired including early ripening, discoloration of the fruit, and a thinner pericarp. (Bemer et al, 2012; Wang et al, 2014). These findings suggest that the differences in FUL2 function play a significant role in distinguishing dry and fleshy fruit development in Solanaceae. Taking in consideration that three NobFUL genes were isolated from $N$. Obtisfulia (data not shown). Functional study of all three genes would reveal additional information about floral transition and fruit development. At the end it may lead to novel genetic technologies for modification of FUL to develop more economically desirable fruits.

\section{REFERENCES}

Aly R, Cholakh H, Joel DM, Leibman D, Steinitz B, Zelcer A, Naglis A, Yarden O, Gal-On A 2009. Gene silencing of mannose 6-phosphate reductase in the parasitic weed Orobanche aegyptiaca through the production of homologous dsRNA sequences in the host plant. Plant Biotechnology, 7: 487-498.

Bachan S, Dinesh-Kumar SP. 2012. Tobacco rattle virus (TRV)-based virus-induced gene silencing. Methods In Molecular Biology, 894: 83-92.
Bemer M, Karlova R, Ballester AR, Tikunov YM, Bovy AG, Wolters-Arts M, Rossetto Pde B, Angenent GC, de Maagd RA. 2012. The tomato FRUITFULL homologs TDR4/FUL1 and MBP7/FUL2 regulate ethyleneindependent aspects of fruit ripening. The Plant Cell, 24: 4437-4451.

Burch-Smith T. M., Anderson J C, Martin G B, Dinesh-Kumar S P. 2004. "Applications and advantages of virus-induced gene silencing for gene function studies in plants," The Plant Journal, 39 (5): 734-746.

Burko Y, Shleizer-Burko S, Yanai O, Shwartz I, Zelnik I D, Jacob-Hirsch J, Kela I, EshedWilliams L, Ori N. 2013. A Role for APETALA1/FRUITFULL Transcription Factors in Tomato Leaf Development. The Plant Cell, 25: 2070-2083.

Busi MV, Bustamante C, D'Angelo C, HidalgoCuevas M, Boggio SB, Valle EM, Zabaleta E. 2003. MADS-box genes expressed during tomato seed and fruit development. Journal of Plant Molecular Biology, 52 (4), 801-815.

Chiarini FE, Barboza GE. 2009. Fruit anatomy of species of Solanum sect. Acanthophora (Solanaceae). Flora. 204: 146-156.

Dinesh-Kumar SP, Anandalakshmi R., Marathe R., Schiff M., Liu Y. 2003. "Virus-induced gene silencing" Methods in Molecular Biology, 236: 287-294.

Ding SW, Voinnet O. 2007. Antiviral immunity directed by small RNAs. Cell, 130:413-426.

Dong Y, Burch-Smith TM, Liu Y, Mamillapalli P, Dinesh-Kumar SP. 2007. A LigationIndependent Cloning Tobacco Rattle Virus Vector for High-Throughput Virus-Induced Gene Silencing Identifies Roles for NbMADS4-1 and $\quad-2$ in $\quad$ Floral Development. Plant Physiol. 145(4): 11611170 . 
Ferrándiz C, Liljegren SJ, Yanofsky MF. 2000a Negative regulation of the SHATTERPROOF genes by FRUITFULL during Arabidopsis fruit development. Science, 289 (5478):436-8.

Ferrándiz C, Gu Q, Martienssen R, Yanofsky MF. 2000b. Redundant regulation of meristem identity and plant architecture by FRUITFULL, APETALA1 and CAULIFLOWER. Development, 127(4) :72534.

Ferrándiz C. 2002. Regulation of fruit dehiscence in Arabidopsis. Journal of Experimental Botany 53: 2031-2038.

Ferrándiz C, Fourquin C. 2014. The role of the FUL-SHP network in the evolution of fruit morphology and function. Journal of Experimental Botany 65, 4505-4513.

Giovannoni JJ. 2004. Genetic Regulation of Fruit Development and Ripening. The Plant Cell, 16: S170-S180.

Gu Q, Ferrándiz C, Yanofsky MF, Martienssen R. 1998. The FRUITFULL MADS-box gene mediates cell differentiation during Arabidopsis fruit development. Development 125: 1509-1517.

Jang S, An G. 1999. NsMADS1, a member of the MADS gene family from Nicotiana sylvestris. Journal of Plant Biology, 42: 85-87.

Jang S, Hong MY, Chung YY, An G. 1999. Ectopic expression of tobacco MADS genes modulates flowering time and plant architecture Molecules and Cells. 9(6):576-86

Jang S, An K, Lee S, An G. 2002. Characterization of Tobacco MADS-box Genes Involved in Floral Initiation. Plant and Cell Physiology, 43(2): 230-238.

Kalantidis K, Schumacher HT, Alexiadis T, Helm JM. 2008. RNA silencing movement in plants. Biol Cell, 100: 13-26.
Melzer S, Lens F, Gennen J, Vanneste S, Rohde A, Beeckman T. 2008. Flowering-time genes modulate meristem determinacy and growth form in Arabidopsis thaliana. Nature Genetics, 40: $1489-1492$.

Müller BM, Saedler H, Zachgo S. 2001. The MADS-box gene DEFH28 from Antirrhinum is involved in the regulation of floral meristem identity and fruit development. Plant Journal, 28(2): 169-79.

Ostergaard L, Kempin SA, Bies D, Klee HJ, Yanofsky MF. 2006. Pod shatter-resistant Brassica fruit produced by ectopic expression of the FRUITFULL gene. Plant Biotechnology Journal, 4: 45-51.

Pabon-Mora N, Ambrose BA, Litt A. 2012. Poppy APETALA1/FRUITFULL Orthologs Control Flowering Time, Branching, Perianth Identity, and Fruit Development. Plant Physiology, 158: 1685-1704.

Robertson D. 2004. VIGS vectors for gene silencing: many targets, many tools. Annual Review of Plant Biology, 55: 495-519.

Smykal P, Gennen J, De Bodt S, Ranganath V, Melzer S. 2007. Flowering of strict photoperiodic Nicotiana varieties in noninductive conditions by transgenic approaches. Plant Molecular Biology 65: 233-242.

Soni P, Siddiqui AA, Dwivedi J, Soni V. (2012). Pharmacological Properties of Datura stramonium L. as a Potential Medicinal Tree: An Overview. Asian Pacific Journal of Tropical Biomedicine, 2(12): 1002-1008.

Voinnet O. 2001. RNAi silencing as a plant immune system against viruses. Trends in Genetics, 17: 449-459.

Wang S, Lu G, Hou Z, Luo Z, Wang T, Li H, Zhang JYe. Z. 2014. Members of the tomato FRUITFULL MADS-box family regulate style abscission and fruit ripening. Journal of Experimental Botany, 65 (12): 3005-3014. 
الملخص العربى

FRUITFULL مؤثر على نمو نبات النيكوتين من حيث تعزيز الاتقال الى مرحلة الازهار ولكنه لم يغير تطور الثمار الكابسولية بثكل ملحوظ فى هذا النبات عبير محمد السيد قسم النبات الزراعى - كلية الزر اعثة(سابا بانشا)- جامعة الإسكندرية

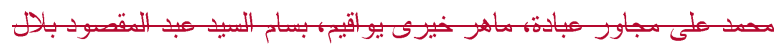

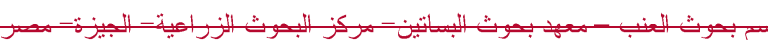

ان فهم آليات الوراثة التى تؤثر على نمو وتطور الازهار والثمار هو أمر بالغ الأهمية للوصول الى بـر امج

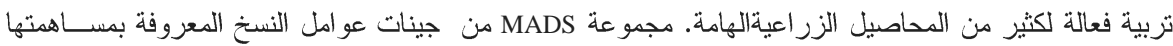

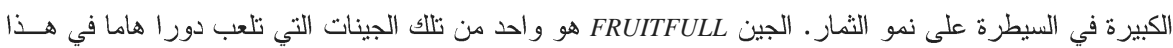

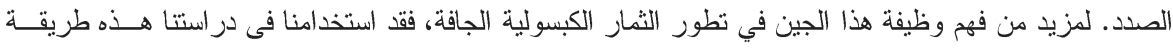

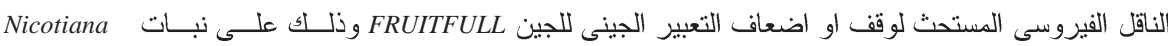
obtusifolia

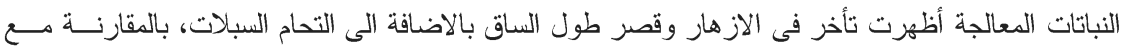

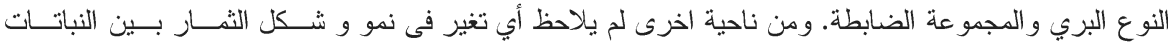

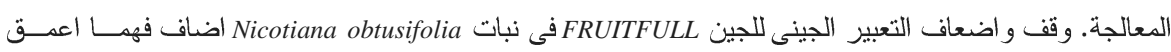

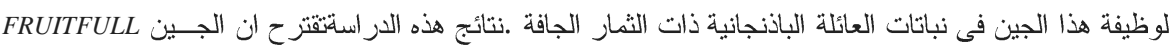
يلعب أدوار امختلفة في تطور الثمار الجافة بالمقارنة مع الثمار اللحمية داخل هذه العائلة. 
